\title{
Dynamic calibration uncertainty of three-axis low frequency accelerometers
}

\author{
Giulio D’Emilia, Antonella Gaspari, Emanuela Natale \\ University of L'Aquila, Dipartimento di Ingegneria Industriale e dell'Informazione e di Economia, Via G. Gronchi 18,67100 L'Aquila, Italy
}

\begin{abstract}
In this paper a methodology concerning the static and dynamic calibration of three-axis low-cost accelerometers in the (0 to 10$) \mathrm{Hz}$ frequency range is described, to be used for evaluation of existing civil infrastructures.

Main and cross sensitivities of the accelerometers have been experimentally estimated by means of the matrix sensitivity concept.

The standard deviation of accelerations obtained along all three axes using different calibration data sets in repeatability conditions has been calculated and intended as dynamic calibration uncertainty.

The method has been validated by using reference accelerations accurately realized, in order to evaluate the residual bias error.

Static and dynamic calibration test benches have been used to realize reference accelerations. In order to create a three-axis acceleration field, a mechanical arm is used in static calibration; a rotary device is used in order to test the accelerometers in dynamic conditions.

According to the procedure described in this paper, a great improvement of the low cost accelerometers' metrological characterization could be achieved, especially in dynamic working conditions.
\end{abstract}

\section{Section: RESEARCH PAPER}

Keywords: Three-axis accelerometer, low frequency vibration, calibration, uncertainty, cross sensitivity

Citation: Giulio D’Emilia, Antonella Gaspari, Emanuela Natale, Dynamic calibration uncertainty of three-axis low frequency accelerometers, Acta IMEKO, vol. 4, no. 4, article 14, December 2015, identifier: IMEKO-ACTA-04 (2015)-04-14

Editor: Paolo Carbone, University of Perugia, Italy

Received December 23, 2014; In final form June 12, 2015; Published December 2015

Copyright: (c) 2015 IMEKO. This is an open-access article distributed under the terms of the Creative Commons Attribution 3.0 License, which permits unrestricted use, distribution, and reproduction in any medium, provided the original author and source are credited

Corresponding author: Giulio D’Emilia, Giulio.demilia@univaq.it

\section{INTRODUCTION}

Social resilience to disasters is now considered a topic of highest political and technical concern in advanced nations.

Social safety and building heritage along with sustainable urban development are important issues in setting guide lines which aim to improve this topic. For this purpose, evaluating the conditions and performance of existing civil infrastructures is a crucial aspect that allows the decision-makers to configure the best lines of activity in order to improve the quality of life [1].

To have integrated procedures involving geotechnical and structural aspects finalized to buildings' diagnostics, a distributed sensor network is needed, allowing us to operate in a selective manner and in the most critical situations. This implies the need of a greater number of measuring sensors, for both the three-axis vibration as well as inclination measurements.
This innovation, together with the need of covering wide areas through this multi-sensors network in order to include different buildings, sets the requirement of lower-cost solutions that are nevertheless able to ensure the requested uncertainty of measurements.

Most of the above mentioned measurement and cost requirements could be satisfied by the well-known microelectro-mechanical systems (MEMS) technology and some examples of its use for these applications can be found in literature [2], even though careful attention should be paid to many possible causes of errors [3], especially when low-cost and low-precision MEMS accelerometers are considered. Due to these facts, many studies can be found in literature, referring to calibration of these sensors, using mechanical reference quantities [3], [4], or using fully electrical methods to estimate the sensitivity of capacitive MEMS accelerometers in batch fabrication [5]. 
Even if different aspects are studied, like bias correction and main and cross sensitivity evaluation, an exhaustive description of calibration uncertainty causes cannot be found, in particular with reference to the dynamic behaviour in the low frequency range (0 to $10 \mathrm{~Hz})$.

Market and literature solutions [2], [6], [7] and characteristics of the calibration test bench designed for this specific application [8], [9], [10], [11], [12], [13], [14] have been considered, but the provided solutions do not appear completely satisfactory if the right trade-off between uncertainty of calibration on one hand, and the cost of the test bench and the calibration procedure duration on the other hand are considered.

In fact, only in a few cases the analysis refers to three-axis accelerometers and the test rigs are generally very expensive; they are based on three-dimensional shakers and only consider the high frequency range [13], [14]. When the low frequency range is examined, different devices have been used [2], [6], [7] [8], [9], [10], [11], [12]; in all these cases, the cross sensitivities are not determined and this appears detrimental with respect to the achievement of a satisfactory sensor accuracy.

Furthermore, bearing in mind these considerations, the first aspect to be considered is the limit that could be achieved with reference to the uncertainty of sensors to be used for acceleration and inclination, taking into account cost requirements as well.

This goal involves sensor behaviour, its power supply and conditioning, data acquisition technique, and initial and periodical calibration, especially in the low frequency range of vibrations (0 to 10$) \mathrm{Hz}$, but calibration requirements appear to be mandatory.

This paper aims to investigate the main technical and procedural aspects to be considered for the definition of a calibration procedure modulated in function of the application, as well as the cost range of the sensors.

Particular attention is paid to the experiment design, the way in which a mechanical reference acceleration is created and evaluated and how data processing techniques influence the variability of results, retrieving useful information without having to perform superfluous tests, according to the quality level of the sensors that are being selected.

It is to be pointed out that straight through the paper the acceleration will be expressed in terms of $g$, acceleration due to gravity; the authors are aware that this is formally incorrect, but they prefer this solution for better understanding of results in the field of civil and seismic engineering.

In section 2 the methodology will be discussed, concerning the experimental solutions able to realize the requested reference acceleration and the procedures for data processing

In section 3 results of tests are presented which have been carried out according to the methodology of section 2 ; the remarkable reduction of systematic errors will be shown, together with the compensation of cross sensitivity effects for measurement uncertainty reduction. The comparison of contributions of different parameters will be used to also identify the specific actions to do for calibration procedure improvement.

\section{METHODOLOGY}

Scientific evaluations, experts' advice and market analysis led us to define the following ranges of interest for tests:

- frequency range: (0 to 40$) \mathrm{Hz}$;
- maximum amplitude of vibration: about $20 \mathrm{~m} / \mathrm{s}^{2}$.

In this paper the calibration in the frequency range $(0-4)$ $\mathrm{Hz}$ will be studied, with the perspective of increasing this range to $10 \mathrm{~Hz}$. For the frequency range $(10-40) \mathrm{Hz}$, other solutions are available, in particular three-axis electro-dynamic shakers.

The three-axis sensor will be calibrated both statically and dynamically. In fact, in field applications we are interested to use it as an inclinometer, the steady behaviour, and as a threeaxis accelerometer, involving the dynamic behaviour.

For the general calibration of the sensor the following relationship between the input acceleration and the output signals can be set:

$\left(\begin{array}{l}a_{x} \\ a_{y} \\ a_{z}\end{array}\right)=\left(\begin{array}{lll}S_{x x} & S_{x y} & S_{x z} \\ S_{y x} & S_{y y} & S_{y z} \\ S_{z x} & S_{z y} & S_{z z}\end{array}\right)^{-1}\left(\begin{array}{c}V_{x} \\ V_{y} \\ V_{z}\end{array}\right)+\left(\begin{array}{l}q_{x} \\ q_{y} \\ q_{z}\end{array}\right)$,

where the matrix $S=S_{i j}$ is the sensitivity matrix, $a=\left(\begin{array}{l}a_{x} \\ a_{y} \\ a_{z}\end{array}\right)$ are the reference acceleration components, $V=\left(\begin{array}{c}V_{x} \\ V_{y} \\ V_{z}\end{array}\right)$ are the voltage outputs of the sensor, and $q=\left(\begin{array}{l}q_{x} \\ q_{y} \\ q_{z}\end{array}\right)$ is the offset vector.

A total of 12 parameters have to be evaluated [3], [13], [14], [15].

Depending on conditions (static or dynamic), static sensitivity matrix $S_{\mathrm{S}}$ and dynamic sensitivity matrix $S_{\mathrm{D}}$, along with their corresponding offset vectors, $q_{\mathrm{s}}$ and $q_{\mathrm{D}}$ respectively, should be evaluated.

For the static calibration a mechanical arm is used in order to adjust the sensor to different positions, through the gravity acceleration vector components along the three axes.

The minimum number of measurement orientations is 4 , although more measurements may give a more robust calibration, using a linear Least Squares Optimization. In this article, the effect of the choice of the number of the angular positioning on the calibration uncertainty is investigated in order to provide useful information about the calibration procedure that is more suitable for the characteristics of the selected accelerometers.

The behaviour of the main and transverse sensitivities in the frequency range (0 to 10$) \mathrm{Hz}$ is also a point of interest.

For the dynamic calibration purpose, a test bench based on a rotary device is used. It is driven by a brushless servomotor, controlled by a Programmable Logic Controller (PLC) by means of a high accuracy angular encoder, which allows us to realize different motion laws (sinusoidal, saw-tooth, ramp, etc..).

The test bench is depicted in Figure 1.

The accelerometer is placed so that $\mathrm{X}-\mathrm{Y}$ or $\mathrm{X}-\mathrm{Z}$ planes do not correspond to the horizontal one, as Figure 2 shows: in this way all the measuring axes will be subjected to a variable acceleration at a frequency depending on the repetition rate of oscillations. In particular:

- the $x$ and $y$ axes measure components (depending on the angle $\alpha$ in Figure 2) of the gravity acceleration $g$ and of the tangential acceleration $a_{\mathrm{t}}$;

- the $z$ axis measures the centripetal acceleration $a_{\mathrm{c}}$.

The tangential acceleration $a_{\mathrm{t}}$ and the centripetal acceleration $a_{\mathrm{c}}$ are defined by the following equations, where $\omega$ is the 


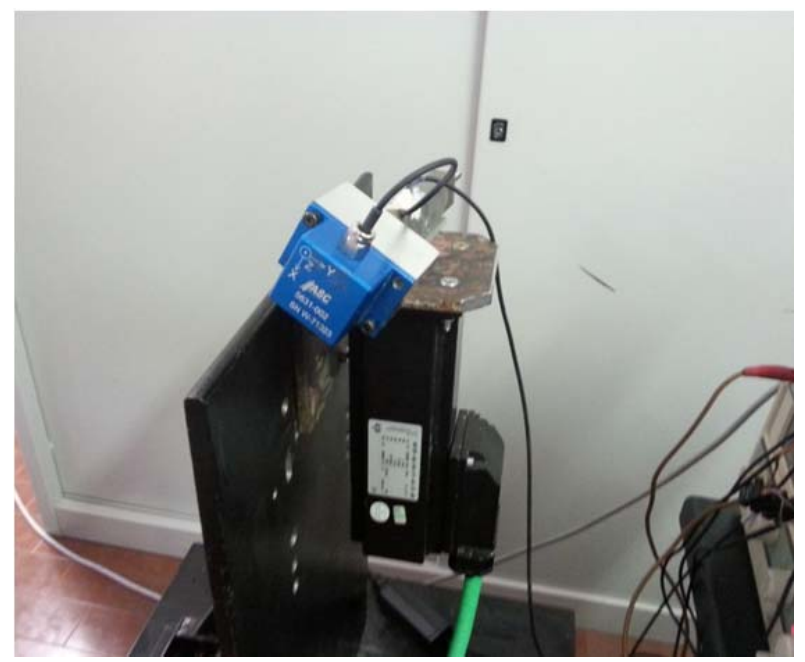

Figure 1 . The rotary table, driven by the brushless motor. Rotation angle is measured by a high accuracy angular encoder.

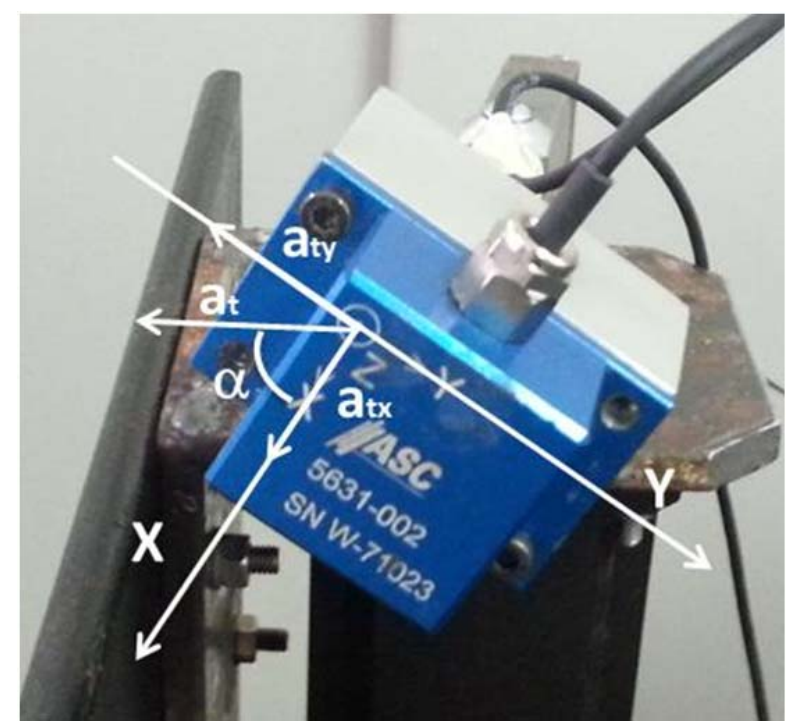

Figure 2. Scheme of tangential acceleration components corresponding to a rotation angle $\alpha$ of the plane $\mathrm{X}-\mathrm{Z}$ with respect to the horizontal one.

angular velocity and $r$ is the distance of the sensitive element of the accelerometer with respect to the axis of rotation:

$a_{t}=\dot{\omega} \cdot r$

$a_{c}=\omega^{2} \cdot r$

In order to build the dynamic sensitivity matrix, a range of different values are assumed for the orientation angle $\alpha$, described in Figure 2.

A high accuracy three-axis accelerometer could also be employed for further validation purposes, according also to literature indications [12].

As to the uncertainty of radius, $r$ (Figure 3), tests are carried out at different radial positions of the sensors, with the purpose of reducing the whole uncertainty on the reference values of acceleration $a_{\mathrm{t}}$ and $a_{\mathrm{c}}$.

In particular, the sensor has been positioned at increasing distances from the axis of rotation using blocks of known thickness, starting from an initial distance, $r_{0}$.

The relationship between the output of the sensor, $y$, and the increase $\Delta r$ of the distance from the rotation axis with respect to the initial distance, $r_{0}$, has been linearly fitted.

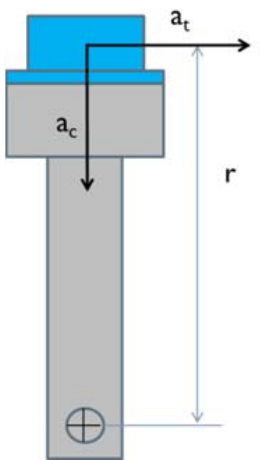

Figure 3. Distance $r$ of the measuring point from the centre of rotation.

The equation of the straight line has been obtained by a least squares linear fitting:

$y=m \cdot \Delta r+b$ and $r=b / m$.

The graph in Figure 4 shows the experimental relationship between the output of the sensor and the increase $\Delta r$ of the distance from the rotation axis.

The distance $r$ of the measurement point of the accelerometer has been obtained according to (2), being 112.7 $\mathrm{mm}$.

The standard uncertainty of $r, s_{\mathrm{r}}$, can be evaluated, according to the following equation [16]:

$s_{r}=\sqrt{\left(\frac{1}{m} \cdot s_{b}\right)^{2}+\left(-\frac{b}{m^{2}} \cdot s_{m}\right)^{2}}$,

where $b$ and $m$ are the slope and the intercept of equation (2), obtained by a least squares method interpolation.

The uncertainty contributions related to $b$ and $\mathrm{m}, s_{\mathrm{b}}$ and $s_{\mathrm{m}}$ respectively, can be calculated according to the following equations [17]:

$s_{b}^{2}=s_{V}^{2} \sum \Delta r_{i}^{2} /\left(N \sum \Delta r_{i}^{2}-\left(\sum \Delta r_{i}\right)^{2}\right)$

$s_{\mathrm{m}}^{2}=N s_{\mathrm{V}}^{2} /\left(N \sum \Delta r_{\mathrm{i}}^{2}-\left(\sum \Delta r_{\mathrm{i}}\right)^{2}\right)$

where:

$s_{\mathrm{V}}^{2}=[1 /(N-2)] \sum\left(m \Delta r_{\mathrm{i}}+b-V_{\mathrm{i}}\right)^{2}$.

$\Delta r_{1}$ are the input values of the increase $\Delta r$, and $V_{\mathrm{i}}$ are the corresponding output tension values of the accelerometer.

From experimental data results: $s_{\mathrm{V}}=3.2 \cdot 10^{-4} \mathrm{~V}$, $s_{\mathrm{b}}=2.6 \cdot 10^{-4} \mathrm{~V}$ and $s_{\mathrm{m}}=1.2 \cdot 10^{-5} \mathrm{~V} / \mathrm{mm}$.

By substituting the relevant values obtained from the experimental test into (5), the relative uncertainty of $r$ is in the order of $0.5 \%$, which is satisfactory.

The effect of the actual shape of motion has to be analysed
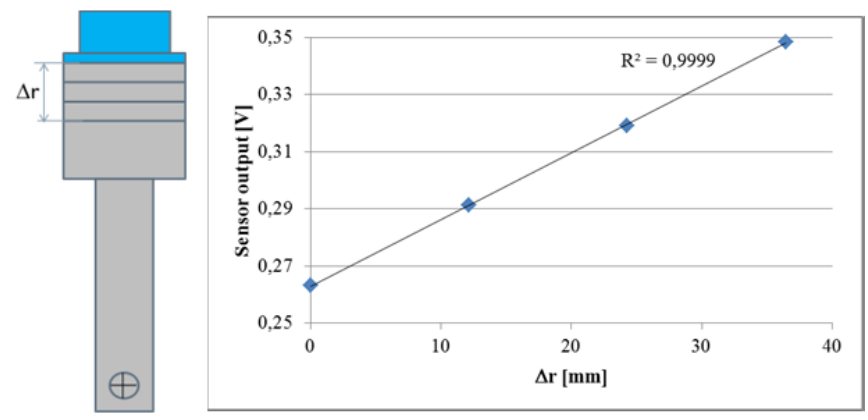

Figure 4. Method for the determination of the distance $r$. 
too, in relation to its theoretical setting, as well as the rotary device set-up. In particular the behaviour of the calibration bench is examined in case of both square wave and sinusoidal motion law for periodical oscillations.

The whole uncertainty of $a_{\mathrm{c}}$ and of $a_{\mathrm{t}}$ will be evaluated, taking into account the repeatability standard deviation of them, in correspondence of each angular position and the standard uncertainty of $r$. The absence of systematic effects on the angular position will be verified.

Tests have been planned in order to consider the following aspects with reference to the uncertainty of $a_{\mathrm{c}}$ and of $a_{\mathrm{t}}$ (or a component of them depending on the position):

- motion laws;

- rotary device setting;

- choosing of the reference;

- number of points for calibration.

Finally it is to be pointed out that the requested calibration procedure and the accuracy to be achieved should be able to fit the sensor characteristics, so that the performances of calibration rig and cost of calibration can be adequately reduced.

\section{RESULTS}

\subsection{Static calibration}

As a first step, a static calibration of a three-axis accelerometer has been done, according to the procedure described in [14], based on (1).

In order to obtain a more robust calibration with respect to the case when the minimum necessary number of positions is used being equal to 4 , a total of 10 different angular positions have been realized.

The results are described in the following static sensitivity matrix:

$S_{\mathrm{S}}=\left(\begin{array}{ccc}-9.863 & -0.006070 & -0.04011 \\ -0.1964 & -9.936 & -0.03548 \\ 0.7081 & 0.03142 & -9.863\end{array}\right) \mathrm{V} /\left(\mathrm{m} / \mathrm{s}^{2}\right)$

and the offset vector:

$q_{\mathrm{S}}=\left(\begin{array}{l}24.32 \\ 24.03 \\ 26.68\end{array}\right) \mathrm{m} / \mathrm{s}^{2}$.

For geometrical angles a maximum deviation of $1^{\circ}$ has been assumed.

A maximum relative deviation from the mean values of $2 \%$ has been evaluated for the coefficients of the main sensitivities, taking into account the angle deviation.

Anyway, measurements have been carried out after calibration in the range $\pm 9.807 \mathrm{~m} / \mathrm{s}^{2}( \pm \pi / 2$ as for the angle) for each axis, using the local gravity as the source: a maximum deviation of $0.098 \mathrm{~m} / \mathrm{s}^{2}$ has been evaluated. This result suggests that the sensitivity matrix should be evaluated on the whole, due to the compensation effect of the variability of the different matrix coefficients between each other. In fact, each acceleration component is corrected by using a combination of three matrix coefficients; therefore the acceleration variability should be reduced with respect to the coefficient one, if casual effects are considered.

\subsection{Dynamic calibration}

\subsubsection{Identification of the motion law}

In case of dynamic calibration of accelerometers, as a preliminary matter, the effect of the motion time law for oscillations on results accuracy has been investigated: in particular saw tooth and sinusoidal velocity profiles of oscillations were considered.

At a preliminary evaluation a saw tooth velocity profile, that means square wave profile for $a_{\mathrm{t}}$, seemed interesting, having a plateau in $a_{\mathrm{t}}$, being very simple to realize on the test bench from the point of view of PLC programming, and making able to investigate in the same test many frequencies (the odd harmonic components).

This motion law has been discarded, since experimental tests indicated that it is difficult to be realized not only practically but also theoretically, being necessary specific settings, which are meaningless from a physical point of view.

Furthermore, oscillations in the test bench arise when $a_{\mathrm{t}}$ is changed, which is a problem also for sensors to be calibrated.

All the results hereinafter refer to tests driven with a sinusoidal motion law. Tests have been carried out at an oscillation frequency of $0.5,1.0$ and $1.6 \mathrm{~Hz}$; in particular the indication will be stressed referring to $1.6 \mathrm{~Hz}$ oscillation, whose vibration peaks are the highest.

\subsubsection{Identification of the reference}

In a calibration procedure the definition of the reference is a fundamental issue.

The possible alternatives that have been considered are:

- using a reference accelerometer. It is to be noticed that using a high accuracy accelerometer as the reference for accelerometer calibration introduces problems deriving from the different radial positioning of the reference accelerometer with respect to the other one; this aspect is expected to increase the calibration uncertainty of accelerometers;

- using the output signal from the encoder. In particular, the tangential acceleration at and the centripetal one ac can be obtained according to (2) and (3), where $\omega$ is the angular velocity, by differentiating the signal of the angular encoder. The reference for the $\mathrm{z}$-axis coincides with the ac value, while the references for the $\mathrm{x}$ - and $\mathrm{y}$ axes coincide with the sum of the appropriate components of the gravity acceleration $g$ and of the tangential acceleration at, depending on the sensor orientation (Figure 2);

- using the motion law that manages the motion. This means that the angular velocity and the acceleration at different instants, according to (2) and (3), are obtained not from the real signal of the encoder, but from the theoretical angular velocity, set by the PLC.

The graphs of Figure 5 show a comparison between real and theoretical time behaviour of the acceleration components, with reference to the axes of the accelerometer. It is to be pointed out that the components of $a_{\mathrm{t}}$ act along the $\mathrm{X}$ - and $\mathrm{Y}$-axes, while $a_{\mathrm{c}}$ acts along the $\mathrm{Z}$-axis.

It can be noticed from the graph of Figure 5 that the systematic error of the reference signal on the whole is negligible; in fact, the reference real signal oscillates around the theoretical one, without any offset.

The Root Mean Square (RMS) of the difference between real $a_{\mathrm{xr}}(t), a_{\mathrm{yr}}(t), a_{\mathrm{zr}}(t)$ and theoretical values $a_{\mathrm{xt}}(t), a_{\mathrm{yt}}(t), a_{\mathrm{zt}}(t)$, during the oscillation period, has been evaluated, being $0.28 \mathrm{~m} / \mathrm{s}^{2}, 0.50$ $\mathrm{m} / \mathrm{s}^{2}$ and $0.12 \mathrm{~m} / \mathrm{s}^{2}$ respectively.

Obviously, most of the oscillations found in $a_{\mathrm{xr}}(t)$ and $a_{\mathrm{yr}}(t)$ depends on numerical differentiation, even though higher order harmonics can be identified with respect to the theoretical 


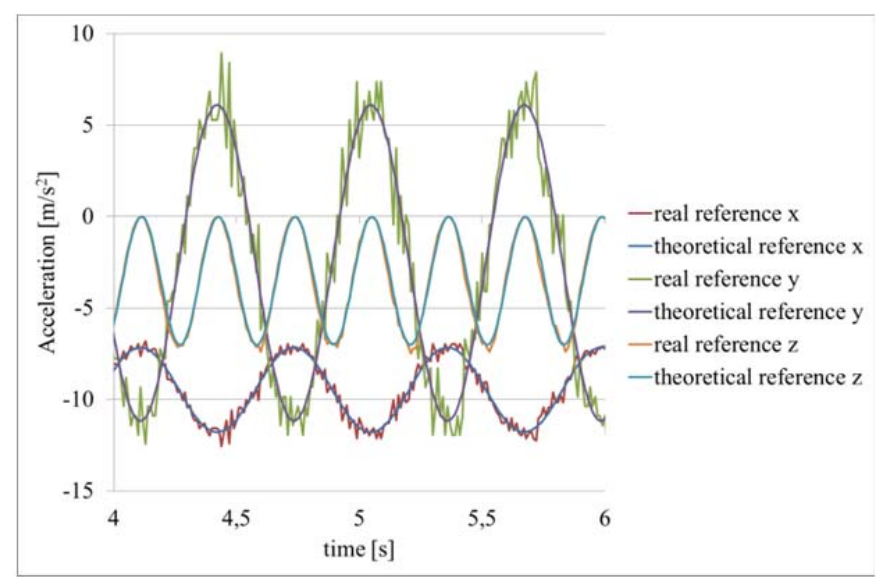

Figure 5. Comparison between real and theoretical time behaviour of the acceleration components, with reference to the axes of the accelerometer.

behaviour, due to effective oscillations at points where the motion is inverted.

Anyway, if the RMS is evaluated of the differences between output of the sensor corrected by the "theoretical" matrix (the matrix $S_{\mathrm{D}}$ obtained by using the theoretical reference) and the real reference, the following value are obtained: $R M S_{\mathrm{x}}=0.36$ $\mathrm{m} / \mathrm{s}^{2} ; R M S_{\mathrm{y}}=1.3 \mathrm{~m} / \mathrm{s}^{2} ; R M S_{\mathrm{z}}=0.35 \mathrm{~m} / \mathrm{s}^{2}$

However, the RMS of the differences between output of the sensor corrected by the "real" matrix (the matrix $S_{\mathrm{D}}$ obtained by using the real reference) and the real reference are: $R M S_{\mathrm{x}}=$ $0.35 \mathrm{~m} / \mathrm{s}^{2} ; R M S_{\mathrm{y}}=1.2 \mathrm{~m} / \mathrm{s}^{2} ; R M S_{\mathrm{z}}=0.32 \mathrm{~m} / \mathrm{s}^{2}$.

A number $n=20$ of points during the cycle were used to evaluate the matrix $S_{\mathrm{D}}$ : the best choice for $n$ will be discussed hereinafter.

Then, a slight improvement using the real reference for the calculation of the matrix is observed, despite the noise produced by the numerical differentiation, since only the real reference can see all the actual vibrations of the calibration bench. For these reasons, the real reference, obtained from the real encoder signal, has been chosen.

\subsubsection{Number of points for calibration and preliminary considerations}

In Table 1 the Root Mean Square of the differences between reference acceleration and corrected sensor data during the oscillation period are evaluated, as a function of the number of points $n$. In Table 1 reference data are those obtained by twice differentiation of the angular encoder data. The effect of building sensitivity matrix with an increasing number of points, $n$, is studied.

Furthermore, provided that a sufficient number of points is considered, no effect of increasing it has been acknowledged. It has been set $n=20$.

The tests so far carried out also suggested some actions to be carried on to improve the calibration procedure

Table 1. RMS of the differences between the output corrected by the dynamic sensitivity matrix and the reference, as a function of $n$.

\begin{tabular}{cccc}
\hline $\boldsymbol{n}$ & $R M S_{\mathrm{x}}\left[\mathrm{m} / \mathrm{s}^{2}\right]$ & $R M S_{\mathrm{y}}\left[\mathrm{m} / \mathrm{s}^{2}\right]$ & $R M S_{z}\left[\mathrm{~m} / \mathrm{s}^{2}\right]$ \\
\hline 14 & 0.762 & 1.12 & 0.327 \\
21 & 0.395 & 1.10 & 0.293 \\
28 & 0.381 & 1.07 & 0.288 \\
35 & 0.379 & 1.03 & 0.287 \\
42 & 0.392 & 1.08 & 0.292
\end{tabular}

performances by reducing calibration uncertainty, in particular of $a_{\mathrm{t}}$ and its components; this objective will be pursued by making more accurate and repeatable the real reference acceleration profile.

These actions on one hand require a mechanical improvement of the rotary table, allowing us to increase the transferability of the angular encoder information to the position of the accelerometer to be calibrated, on the other hand ask for a higher sampling rate of the angular encoder data.

It is to be pointed out that the used PLC allows us to use a task time of less than $1 \mathrm{~ms}$, allowing a sampling rate of about $1 \mathrm{kHz}$.

Rather than increasing the number of points on which the sensitivity matrix should be evaluated but provided that a sufficient number of points is considered, it seems more convenient to mix data measured changing the sensor orientation, also in the dynamic calibration.

\subsubsection{Results of the dynamic calibration and uncertainty evaluation}

Considering the observations made in previous paragraphs, 20 different positions of the sensor have been taken into account during every cycle; for each position the reference accelerations along the $\mathrm{x}-, \mathrm{y}$ - and $\mathrm{z}$-axes have been calculated, depending on gravity and motion acceleration components and compared with the corresponding outputs of sensors in all directions.

Then, the matrix $S_{\mathrm{D}}$ and the offset vector $q_{\mathrm{D}}$ in the dynamic case have been calculated on the basis of the reference values, being:

$S_{\mathrm{D}}=\left(\begin{array}{ccc}9.948 & 0.2576 & -0.3657 \\ -0.5342 & 10.97 & -0.6611 \\ -0.4454 & 1.614 & 10.83\end{array}\right) \mathrm{V} /\left(\mathrm{m} / \mathrm{s}^{2}\right)$

$q_{\mathrm{D}}=\left(\begin{array}{l}\mathrm{q}_{\mathrm{x}} \\ \mathrm{q}_{\mathrm{y}} \\ \mathrm{q}_{\mathrm{z}}\end{array}\right)=\left(\begin{array}{l}24.02 \\ 20.10 \\ 26.68\end{array}\right) \mathrm{m} / \mathrm{s}^{2}$

The improvement of using the dynamic sensitivity matrix with respect to the static one is clearly shown in diagrams of Figures 6a, 6b, 6c, where real encoder data, twice differentiated, used as the reference, sensor data corrected by static sensitivity matrix and data corrected by dynamic sensitivity matrix are compared.

A quantitative evaluation of the RMS of the differences between the output corrected of the accelerometer and the reference confirm this observation (Tables 2 and 3).

Table 2. RMS of the differences between the output corrected by the dynamic sensitivity matrix and the reference.

\begin{tabular}{ccc}
\hline $\begin{array}{c}\text { Absolute } \\
\mathrm{RMS}_{\mathrm{x}}\left[\mathrm{m} / \mathrm{s}^{2}\right]\end{array}$ & $\begin{array}{c}\text { Absolute } \\
\mathrm{RMS}_{\mathrm{y}}\left[\mathrm{m} / \mathrm{s}^{2}\right]\end{array}$ & $\begin{array}{c}\text { Absolute } \\
\mathrm{RMS}_{\mathrm{z}}\left[\mathrm{m} / \mathrm{s}^{2}\right]\end{array}$ \\
\hline 0.35 & 1.2 & 0.32 \\
\hline $\begin{array}{c}\text { Relative } \\
\mathrm{RMS}_{\mathrm{x}}\end{array}$ & $\begin{array}{c}\text { Relative } \\
\mathrm{RMS}_{\mathrm{y}}\end{array}$ & $\begin{array}{c}\text { Relative } \\
\mathrm{RMS}_{\mathrm{z}}\end{array}$ \\
\hline 0.15 & 0.14 & 0.09
\end{tabular}

Table 3. RMS of the differences between the output corrected by the static sensitivity matrix and the reference.

\begin{tabular}{ccc}
\hline $\begin{array}{c}\text { Absolute } \\
\mathrm{RMS}_{\mathrm{x}}\left[\mathrm{m} / \mathrm{s}^{2}\right]\end{array}$ & $\begin{array}{c}\text { Absolute } \\
\mathrm{RMS}_{\mathrm{y}}\left[\mathrm{m} / \mathrm{s}^{2}\right]\end{array}$ & $\begin{array}{c}\text { Absolute } \\
\mathrm{RMS}_{\mathrm{z}}\left[\mathrm{m} / \mathrm{s}^{2}\right]\end{array}$ \\
\hline 0.43 & 1.3 & 1.1 \\
\hline $\begin{array}{c}\text { Relative } \\
\mathrm{RMS}_{\mathrm{x}}\end{array}$ & $\begin{array}{c}\text { Relative } \\
\mathrm{RMS}_{\mathrm{y}}\end{array}$ & $\begin{array}{c}\text { Relative } \\
\mathrm{RMS}_{\mathrm{z}}\end{array}$ \\
\hline 0.19 & 0.14 & 0.30
\end{tabular}



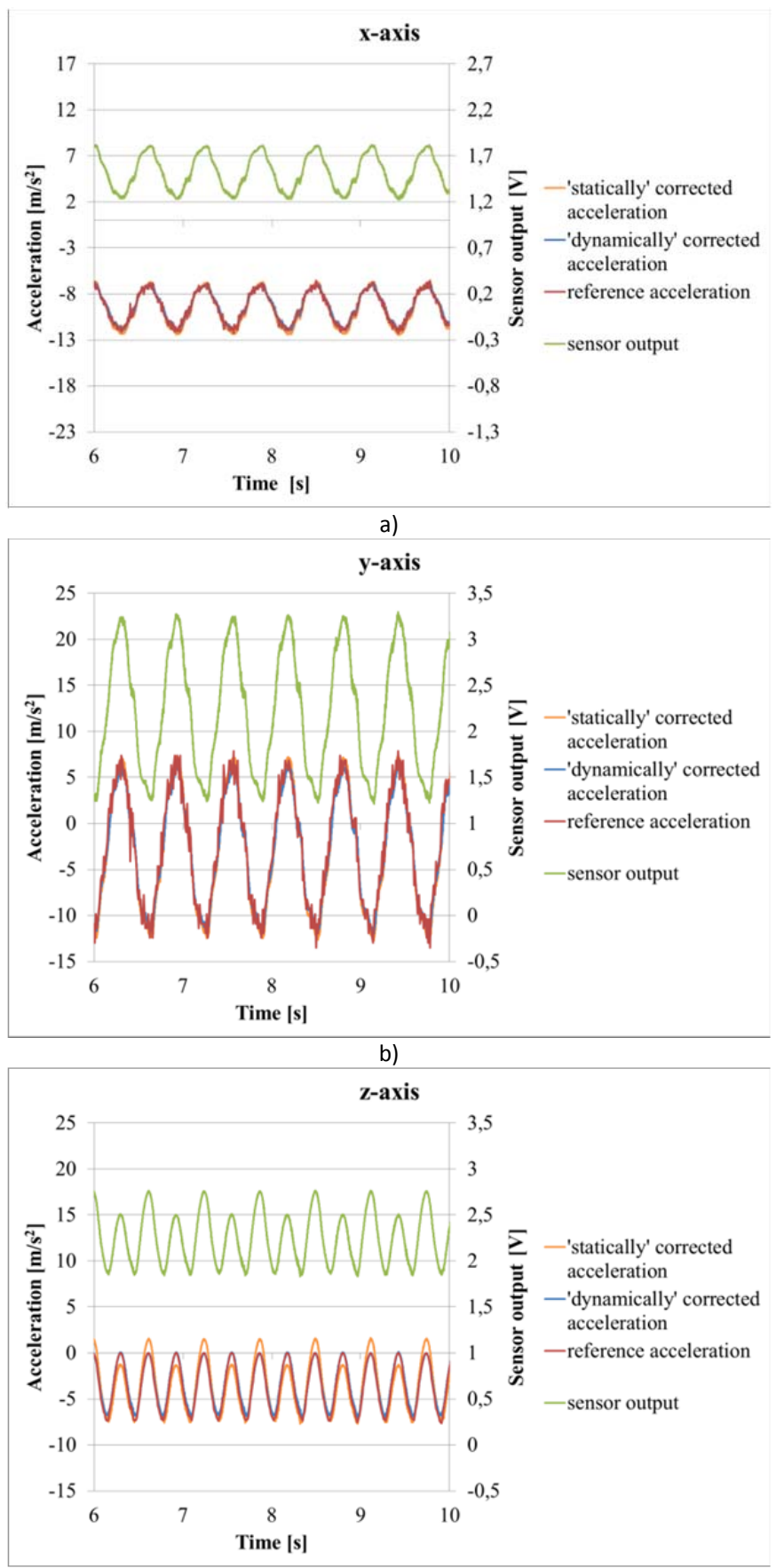

c)

Figure 6. Comparison among real encoder data, twice differentiated, used as the reference, sensor data corrected by static sensitivity matrix and data corrected by dynamic sensitivity matrix. a) $\mathrm{X}$-axis acceleration; b) $\mathrm{Y}$-axis acceleration; c) Z-axis acceleration.

In order to evaluate the variability of the sensor output corrected by the results of the dynamic calibration, 10 different sets of 20 points have been considered, corresponding to different oscillation periods; 10 different matrices $S_{\mathrm{D}}$ and offsets $q_{\mathrm{D}}$ have been correspondingly calculated.

Using them to correct the sensor output during a cycle, the variability is found to be $0.15 \mathrm{~m} / \mathrm{s}^{2}, 0.29 \mathrm{~m} / \mathrm{s}^{2}, 0.071 \mathrm{~m} / \mathrm{s}^{2}$ for the $\mathrm{X}$-axis, $\mathrm{Y}$-axis and $\mathrm{Z}$-axis respectively. The same values, expressed as a percentage of the signal amplitude, are: $6.3 \%$, $3.4 \%$ and $2.0 \%$ respectively. These values can be assumed as standard percentage calibration uncertainties for the $\mathrm{X}$-axis, $\mathrm{Y}$ axis and $Z$-axis accelerations.
The contribution of the uncertainty of the radius $r$ appears to be negligible in any case, being in the order of $0.5 \%$.

If the variability of the reference is considered too, using 10 cycles of the reference signal (Figure 7) an average standard deviation has resulted, equal to: $0.28 \mathrm{~m} / \mathrm{s}^{2}, 1.1 \mathrm{~m} / \mathrm{s}^{2}, 0.16 \mathrm{~m} / \mathrm{s}^{2}$, for the $\mathrm{X}$-axis, $\mathrm{Y}$-axis and $\mathrm{Z}$-axis respectively. These values, expressed as a percentage of the signal amplitude, become: $12 \%, 12 \%, 4.5 \%$ respectively.

Using the sensitivity matrix allows to get a corrected output with a reduced variability with respect to the cycle variations of the reference. This result suggests that improving the cycle repeatability of the reference could further improve the correction and reduce uncertainty of the calibrated sensor.

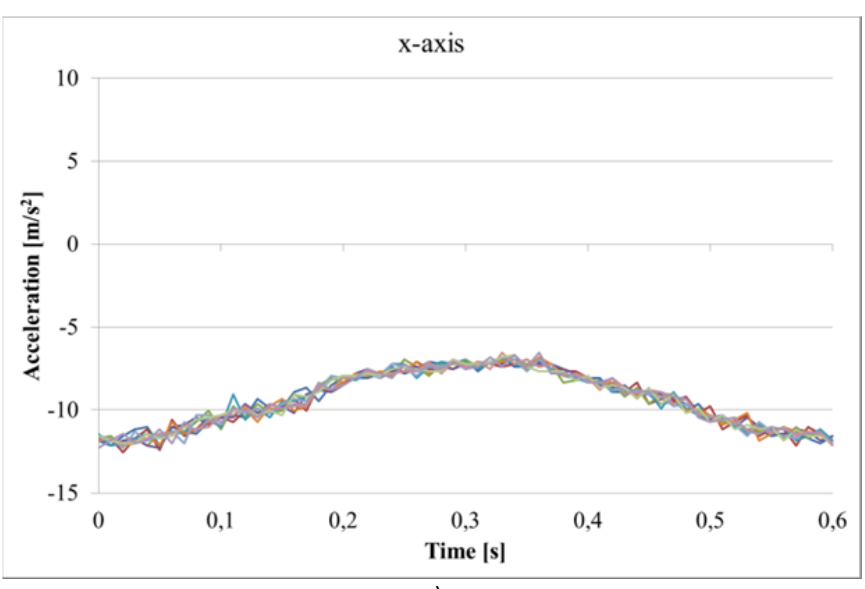

a)

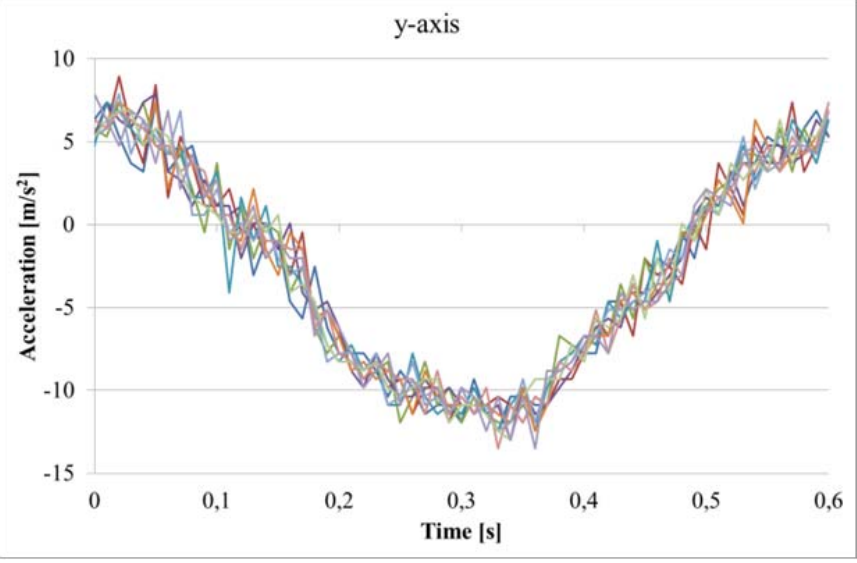

b)

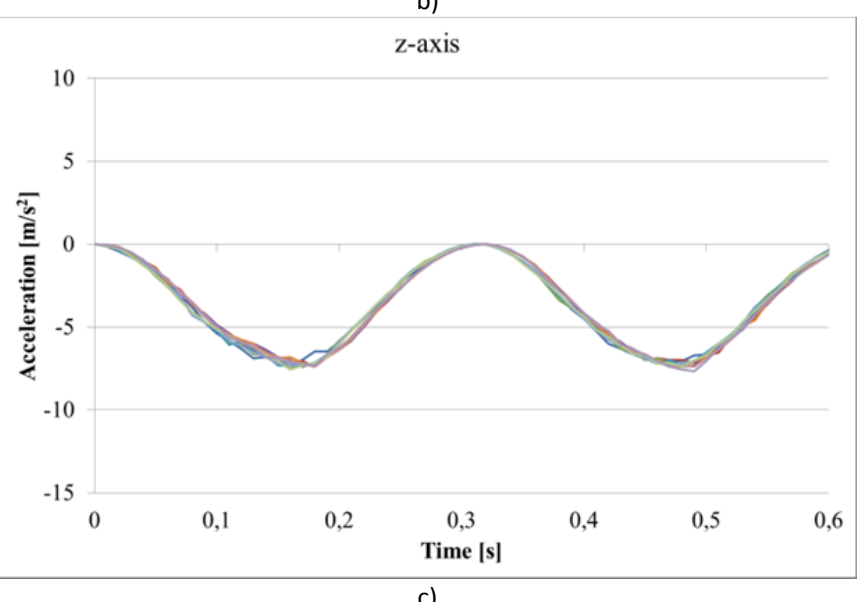

Figure 7. Variability of 10 cycles of the reference acceleration. a) X-axis acceleration; b) Y-axis acceleration; c) Z-axis acceleration. 


\section{CONCLUSIONS}

In this paper some aspects concerning the calibration uncertainty of three-axis low-cost accelerometers for diagnostics of civil buildings are considered. Both static conditions and dynamic behaviour in the range (0 to 4$) \mathrm{Hz}$ as for vibrations have been examined.

A frequency varying three-axis field of acceleration has been realized by modulating both gravity acceleration, and tangential/radial acceleration components of a rotary motion created on a test bench driven by a brushless servomotor.

Reference values for $a_{\mathrm{c}}$ and $a_{\mathrm{t}}$ acceleration (or a component of it depending on position) have been deduced from the indications of a high precision rotary encoder used for axis control, in order to separate the effect on the whole uncertainty of calibration of the test bench characteristics and settings.

The effects taken into account refer to :

- motion laws;

- rotary device setting;

- choosing of the reference;

- number of points for calibration.

Experimental results, for both static and dynamic applications, show that both main and cross sensitivities of a three-axis capacitive accelerometer of good quality level from a metrological point of view can be obtained; this allows to correct the effect of cross sensitivities also in dynamic applications, in order to remarkably improve the transducer accuracy for in field applications.

Using the sensitivity matrix allows to get a corrected output with a reduced variability with respect to the cycle variations of the reference. This result suggests that improving the cycle repeatability of the reference could further improve the correction and reduce uncertainty of the calibrated sensor.

Therefore, the main actions to be realized should move to improve the mechanical behaviour of the test bench, and the acquisition of the angular encoder data. Improving these aspects is expected to further reduce the calibration uncertainty, and also to extend the calibration range up to $10 \mathrm{~Hz}$.

\section{REFERENCES}

[1] Wyss M and Rosset P "Mapping sismic risk: the current crisis" Nat. Haz. 68 (2013) pp. 49-52.

[2] Zhao M and Xiong X "A New MEMS Accelerometer Applied in Civil Engineering and its Calibration Test" The Ninth International Conference on Electronic Measurement \& Instruments ICEMI-2, 2009, pp.122-125.
[3] Ren Wei, Zhang Tao, Zhang Hai-yun, Wang Lei-gang, Zhou Yong-jie, Luan Meng-kai, Liu Hui-feng, Shi Jing-wei "A research on calibration of low-precision MEMS inertial sensors", 25th Chinese Conference on Control and Decision Conference (CCDC), 2013, pp. $3243-3247$

[4] Zanjani P N, Abraham A A, "A Method for Calibrating Micro Electro Mechanical Systems Accelerometer for Use as a Tilt and Seismograph Sensor" 12th International Conference on Computer Modelling and Simulation (UKSim), 2010, pp. 637 641

[5] Dumas N, Azais F, Mailly F, Nouet P, "Study of the electrical set-up for capacitive MEMS accelerometer test and calibration", Journal of Electronic Testing 26 (2010) pp. 111-125.

[6] Milligan D J, Homeijer B D and Walmsley R G "An ultra-low noise MEMS accelerometer for seismic imaging" Sensors (IEEE), 2011, pp. 1281 - 1284.

[7] Vallet F and Marcou J "A low-frequency optical accelerometer" J. Opt. 29 (1998) pp. 152-155.

[8] Schiefer M I and Bono R "Improved low frequency accelerometer calibration" XIX IMEKO World Congress Fundamental and Applied Metrology, 2009

[9] Choi K, Jang S and Kim Y "Calibration of Inertial Measurement Units Using Pendulum Motion" Int. J. of Aeronautical \& Space Sci. 11(3) (2010) pp.234-239.

[10] Zhu C, Qin X, Wu Z and Zheng L "Research on calibration for measuring vibration of low frequency" International Conference on Mechanic Automation and Control Engineering (2010) pp. 3315 - 3318.

[11] Mohd-Yasin F, Nagel D J, Ong D S, Korman C E and Chuah H $\mathrm{T}$ "Low frequency noise measurement and analysis of capacitive micro-accelerometers" Microelectronic Engineering 84 (2007) pp. 1788-1791.

[12] Ohm W, Wu L, Hanes P and Wong G S K “Generation of lowfrequency vibration using a cantilever beam for calibration of accelerometer" Journal of Sound and Vibration 289 (2006) pp. 192-209.

[13] Akira Umeda, Mike Onoe, Kohji Sakata, Takehiro Fukushia, Kouichi Kanari, Hiroshi Iioka, Toshiyuki Kobayashi "Calibration of three-axis accelerometers using a three-dimensional vibration generator and three laser interferometers" Sensors and Actuators A: Physical 114 (2004) pp. 93-101.

[14] Nakano A, Hirai Y, Sugano K, Tsuchiya T, Tabata O, Umeda A, "Rotational motion effect on sensitivity matrix of MEMS threeaxis accelerometer for realization of concurrent calibration using vibration table", IEEE 26th International Conference on Micro Electro Mechanical Systems (MEMS), 2013, pp. 645 - 648.

[15] Mark Pedley, "High Precision Calibration of a Three-Axis Accelerometer" Freescale Semiconductor (2013).

[16] Giulio D'Emilia, Antonella Gaspari, Emanuela Natale, "Evaluation of aspects affecting measurement of three-axis accelerometers", Measurement 77 (2016) pp. 95-104.

[17] Ernest O. Doebelin, Measurement systems, McGraw-Hill , 2004, ISBN 0-07-243886-X. 